\title{
An established practice in new surroundings: concepts, challenges, pitfalls and guidelines for NCHD Balint Groups
}

\author{
L. Douglas ${ }^{1, *}$ and L. Feeney ${ }^{2}$ \\ ${ }^{1}$ Department of Old Age Psychiatry, Tallaght Hospital. Dublin, Ireland \\ ${ }^{2}$ Cluain Mhuire Mental Health Service, Blackrock, Co. Dublin, Ireland
}

Balint groups are now mandatory for psychiatry trainees. Balint groups have been in existence in General Practice for several decades. Providing Balint groups for Psychiatry Non Consultant Hospital Doctors brings with it challenges for the group leader and participants. Many of these challenges are common place in any form of group work, while others are unique to this cohort. This article describes these challenges. Guidelines which offer the the group the best chance of success, in the face of these common challenges, are discussed.

First published online 1 February 2016

Participation in a reflective practice group has become mandatory for Psychiatry trainees in Ireland (Omer \& McCarthy, 2010; College of Psychiatrists of Ireland, 2014). The most common form of reflective practice group in Medical/Clinical settings use are known as Balint groups. Balint groups are reflective practice groups in which participants have the opportunity to discuss psychological aspects of their relationships with patients in a relaxed, supportive, structured and confidential setting. Balint groups are so-called because the use of these groups to assist doctors in their work was first described by Michael Balint, most notably in his 1957 book, The Doctor, his Patient and the Illness (Balint, 1957). According to Balint, the purpose of these groups is 'to examine the relationship between the doctor and the patient to look at the feelings generated in the doctor as possibly being part of the patient's world and then use this to help the patient. If these feelings do not seem to belong to the patient but to the doctor it helps to know that too, to be a participant in a relationship and its observer is fraught with difficulties and potential bias. The aim is to study this (bias) carefully. As a consequence, the doctors can take the feelings that arise from their work seriously and pay attention to much that would otherwise be disregarded.

Although Balint groups were initially devised for GPs, they are now widely used with other groups of health professionals (The Balint Society). Such groups have been long used in some centres as part of psychiatric training. Fitzgerald \& Hunter (2003) Balint groups can provide a meaningful addition to a psychiatry trainee's understanding of their interactions with patients and

* Address for correspondence: Dr Leonard Douglas, Tallaght Mental Health Service, Tallaght Hospital, Dublin 24, Ireland.

(Email: leonarddouglas@yahoo.com) their personal responses to differing clinical situations (Das et al. 2003). Furthermore, Balint groups can aid the generation of perspectives on 'difficult', 'stifling', 'confusing' and 'stuck' interactions. This culminates, one hopes, in a broadening, rather than a narrowing (formulation and diagnosis), of perspective on a particular doctor patient interaction. Such methodologies attend to the dynamics of transference and countertransference within the doctor patient dynamic. As such, issues of power come to the foreground and in so doing the clinician can more readily identify and locate themselves within the transferential matrix of the consultation.

Since the recent mandatory requirements to establish reflective practice groups for Irish psychiatry trainees, Balint groups have been widely established in training centres. Enthused leaders offer their time and trainees are compelled to attend. In practice these groups often struggle to achieve their objectives. Below we examine the many issues that can make or break such a group. This paper was written by the authors following attendance at a Balint group leaders course organised by the Irish division of the Balint Society. It is informed predominantly by the content of group discussions on that day and subsequent reading and experience of the authors (Balint Group Leaders Course: Athlone, 2014).

\section{The ideal}

The typical format for a Balint group usually starts with trainees being asked to consider a suitable case to present. One trainee presents a summary of the case in 5-10 minutes. The group is then invited to ask a few clarifying questions of fact pertaining to the case. Then the presenter sits back and for 20-30 minutes takes no part in the discussion. The group are then encouraged 
to reflect on what could be going on here for the doctor and patient. The leader(s) probes and encourages trainees to imagine, postulate and speculate on any facet of the case. The presenter is then invited back into the group discussion and invited to contribute, only if they wish to, for the final few minutes.

During this process trainees are essentially soaked in a group environment which encourages them to negotiate and explore with curiosity the interpersonal and emotional terrain of doctor-patient relationships without simply reverting to logical thought and intellect (the tried and tested bulwark of doctors). A Balint group may untangle issues pertaining to a case, although often leaving the group members/presenter with more questions than answers. Solutions are actively discouraged by facilitators. This can help the doctor approach the next consultation with the potential to see what 'else' could be present and relevant.

This of course is the ideal scenario. Enlightened trainees and satisfied supervisors eagerly attending a weekly group is not always the reality for many groups.

\section{The reality}

This potential benefit to the trainee can often go unrealised, leaving the group facilitator doubting the value of the process. Often, early into a six month group, participation flags, leaving a motivated facilitator(s) sitting with two or three enthusiastic or obedient trainees at the prescribed time. At other times, in spite of good attendance, there can be a myriad of factors which impede the group from flourishing. Common experiences include: reluctance of trainees to bring a case, group discussion never leaving the logical formulation and treatment territory, generalised discussion about the 'gripes of the job', poor participation of the group members and even outright resistance to the usefulness of group supervision. The enthusiastic facilitator can begin to struggle.

When thinking about why this can happen, and how to improve a Balint group,it is helpful to examine:

1. Issues pertaining to the initial set up/parameters of the group.

Working with groups can be challenging. None more so than a group of non consultant hospital doctors (NCHD's) with varying openness to and experience of group work. Preparatory work done before or early in the group's life, generally reaps rewards. This could include:

- collaborating with group members to agree group rules;

- emphasising confidentiality;

- advocating strongly with colleagues for a suitable time in the weekly schedule when it is understood that all trainees are released from other duties and available to attend.
Over the course of the group being set up the leader should try to share and hand over ownership of the group to the members. At the first meeting of the group it can be helpful to use the full session to describe:

- the purpose of the group and which aspects of doctorpatient interaction it should help to illuminate;

- the format it will take;

- setting up and agreeing ground rules;

- discussion of what it means to be in the group and frank discussion of what can destroy a group;

- issues such as attendance, apologies, late arrivals and resistance to the need for a group in the first place are helpful to name and discuss.

In this the leader is training the trainees in order to prepare them to participate usefully in a process of which they may have varying experiences and preconceptions.

2. Issues pertaining to the facilitator(s).

It is important that the leader can advocate for suitable timetabling to facilitate trainees to attend the group weekly. Following this, if there is more than one leader it can be useful to divide the task of managing the group between them before the group begins. One leader often looks after the detail of the case presented, while the other leader attends to the group's needs. The leader(s) in essence creates a container for the group to operate in.

Below is a list of the ideal characteristics of a group leader(s) (Johnson et al. 2004).

Providing model behaviour

Makes clear interventions

Sits comfortably with uncertainties

Elaborates, reflects, and clarifies thoughts

Elaborates, reflects, and clarifies feelings

Legitimises emotions

Assumes different roles

Empathic with group's process

Creating an Atmosphere of Safety

Sets rules and boundaries as necessary

Protects presenter/members from group crossexamination

Respects all points of view

Provides opportunity for all to speak

Avoids in-depth personal psychological probing

Cultivates a climate of trust 


\section{Observing Group Process}

Intervenes to promote group process

Intervenes appropriately to developmental stage of group

Uses knowledge of parallel process to facilitate group insight

Helps group explore different patient-doctor scenarios

Identifies moods of the group related to the case

Notes themes or topics avoided by group

Each of the above roles could be shared or divided between the leaders.

Leader's interventions often differ. Often a group can engage with one leader's interventions and step back from the others. Sometimes one of the leaders can begin to attack the group. It can take a period of experimentation with different roles for two leaders to find their feet with a group. Allocating regular time each week following the group, for reflection between the leaders can be useful.

While leaders differ it is important that each of the leaders ensures their approach or 'stance' within the group is consistent with guiding principles of leadership of a Balint group. The leader(s) should not take a superior stance, they guide but do not teach. They should attempt to guide the group reaching solutions rather than providing solutions or interpretations. When a leader does disclose their own feelings this should be only in cases where the leader is intervening for a clear reason, for example, to model behaviour appropriate to the group. Rather than challenging the group with direct questions it can be useful for the leader to express their thoughts in the form of a question to themselves (listening to this discussion I'm asking myself ...'). Again the work of the group should left to the group. The leader models good listening, shows empathy and tolerates silence and uncertainity. The leader should have a 'third ear' as to what is 'not being said'; representing the patient or doctor when neglected.

Leaders often report frustration and difficulty with developing reflection within the group during the discussion stage. Consequently, a leader might be tempted to intellectualise or help formulate the case for the trainee at this point. A better approach may be to reflect on parallel processes by encouraging the group to look at what is going on in the group for clues to what may exist in the doctor-patient relationship being discussed. Reflecting on the mood in the group can also loosen the discussion, for example, 'there's a tightness in the air, what is that?' 'does that reflect something in the case?'. In times of difficultly the role of the leader is provide a safe and supportive structure and environment where the doctor-patient relationship can be examined by the group. Moving the focus of the discussion to the doctor or shifting the focus to the patient (e.g. 'what else could be going on for this patient that we don't know about already?') are good examples of having a 'third eye' to what is not being said. Moving the discussion to imaginative rather than concrete/conceptual terms, for example, asking the group to think of images and metaphors that the patient's story conjures up can be a helpful intervention on behalf of the leader.

Sometimes returning to the ground rules and concepts can help remind a group about what is expected. This is particularly relevant in the early stages of the group's life. After all, the group is trying to get in touch with that which is not accessible through logical thought. This requires a culture change for trainees. Leaders may find it helpful to remind themselves on a regular basis to adopt a curious stance to the case (rather than being the 'educator'). This can be liberating for all involved and it models good behaviour to the group members. None of the above should be prescriptive but simply used to reflect on how best to provide flexible, empahtic and supportive guidance for a group.

3. Issues arising within the group members.

There are several recurring issues that arise for group members which can affect the work of the group.

The potential for members to feel resistance to the mandatory nature of the group is worth naming early on with the group. One could reflect to the group that it is mandatory because it matters. This provides an opportunity to describe the important nature of this work. Early acknowledgement of resistance, or other feelings, that are in the room is important. This can enrich the group's discussions, examining how resistance can influence group dynamics and doctor-patient relationships, especially if unexpressed.

When a presenter of a case becomes upset or dysregulated this can be challenging, especially if unexpected.

Patients are constantly pouring their material into the unsuspecting doctor (transference), and the doctor transferring to the patient (countertransference). Why should the doctor be surprised that they carry and ingest patient material at times? An important aspect of the work with group members in this regard is to normalise the fact that we can feel for and be affected by our patients and their lives. Balint groups are important in 'mapping' this process and raising situational awareness around times when our interactions with patients evoke an emotional response. This also has a role in eroding harmful machisms that can be harmful to the doctor as a negative defence mechanism. 
While Balint groups help remind doctors that dealing with the fractured and disturbing stories of patients can be disturbing and upsetting, it is essential that doctors are reminded that from the outset, the group's work is to unravel and create new perspectives on a case rather than being individual/group therapy for a struggling doctor. The idea that the presenter is assumed to be essentially well but needing perspective is critical to impart. This has a two-fold effect:

1. Those who have preconceptions that Balint groups are some form of therapy for the members can be reassured that this is not the case.

2. Members who feel they need individual help are reminded that this is not the forum and to use the group appropriately.

If a member does become upset it is often tempting to try to reassure or intervene after the group. Obviously a leader's job is to protect the presenter from attacks during the group, but once the group is finished it is often the group members who are best placed to support one another. In fact approaching a trainee afterwards uninvited could be shaming. Clear rules instructing members of the group not discuss cases outside of the group without being invited to do so by the presenter are protective to presenters. The leader should be explicit about being open to discussion from trainees but should not usually initiate it.

\section{Issues especially relevant to NCHD Balint groups.}

Late arrivals, clinics running over, trainees not being released, resistance to being involved and annual leave all can contribute to small groups having a varying, almost chaotic, membership each week. When 'selling' the idea of Balint groups to trainees in the first few weeks, it is best to be frank about these issues. NCHD Balint groups are unlikely to be the 'ideal' closed group which meets regularly, developing a trust and confidence between members like Balint described. Being realistic about this and discussing this with the group and asking them how to manage this can help build group cohesiveness. In doing this the leader is slowly handing control and responsibility of the group to the group. The constant changing of colleagues and personnel is a recurring theme in NCHD working lives and can be used to elaborate discussion within the group rather than causing the group to fail. Many leaders have considered augmenting membership with other disciplines. It is hard to imagine that this would help in most cases, particularly given the difficulty NCHDs sometimes experience with selfdisclosure.

In addition, Balint groups are not the panacea for all trainee issues and should not be sold as such.

\section{Conclusion}

The recent establishment of Balint groups for trainees in psychiatry has great potential to illuminate the doctorpatient relationship. This comes with challenges for the participants and leaders of these groups. Leaders can afford a NCHD Balint group the best opportunity to do fruitful work if they can establish a group that encourages trainees to bring a sense of curiosity to cases, trying at all times to leave the work of the group to the group and being aware of the pitfalls with planning and implementation that commonly occur.

Working with patients without support is difficult. With some persistence, reflection and structure, Balint groups can be valuable for many trainees.

\section{Acknowledgements}

The authors thank Patsy Brady and Clive Garland who organised and delivered Balint group training for group leaders in May 2014 in Athlone from which many of the concepts above evolved.

\section{Financial Support}

This research received no specific grant from any funding agency, commercial or not-for-profit sectors.

\section{Conflicts of Interest}

The authors declare no conflicts of interest.

\section{Ethical Standards}

The authors assert that all procedures contributing to this work comply with the ethical standards of the relevant national and institutional committee on human experimentation with the Helsinki Declaration of 1975, as revised in 2008. The authors assert that ethical approval for publication of this audit was not required by their local REC.

\section{References}

Balint M (1957). The Doctor, His Patient and the Illness. Churchill Livingstone: London.

Balint Group Leaders Course (2014). Irish meeting for Balint Group Leaders in association with The Balint Society, UK. Athlone, Ireland.

The Balint Society. Available at http://balint.co.uk/. Accessed 23 November 2014.

College of Psychiatrists of Ireland (2014). Curriculum for basic and higher specialist training in psychiatry. 2 January 2014 (http://www.irishpsychiatry.ie/Libraries/External_Events_ Documents/Curriculum_for_Basic_Higher_Specialist_ Training_in_Psychiatry_Revision_2_January_2014_Amend ments_to_Reflective_Practice_Requirements_Learning Outcomes_Grids.sflb.ashx). Accessed 2 January 2014. 
Das A, Egleston P, El-Sayeh H, Middlemost M (2003). Trainees experiences of a Balint group. Psychiatric Bulletin 27, 274-275.

Fitzgerald G, Hunter MD (2003). Organising and evaluating a Balint group for trainees in psychiatry. Psychiatric Bulletin 27, 434-436.
Johnson AH, Nease DE Jr, Milberg LC, Addison RB (2004). Essential characteristics of effective Balint group leadership. Family Medicine 36, 253-259.

Omer S, McCarthy G (2010). Reflective practice in psychiatric training: Balint groups. Irish Journal of Psychological Medicine 27, 115-116. 